\title{
Time-bounded incompressibility of compressible strings and sequences
}

\author{
Edgar G. Daylight ${ }^{\mathrm{a}, 1}$, Wouter M. Koolen ${ }^{\mathrm{b}}$, Paul M.B. Vitányi ${ }^{\mathrm{b}, \mathrm{c}, *}$ \\ a University of Amsterdam, Institute of Logic, Language, and Computation, Amsterdam, The Netherlands \\ b Centrum voor Wiskunde en Informatica, Science Park 123, 1098 XG Amsterdam, The Netherlands \\ c University of Amsterdam, Department of Computer Science, Amsterdam, The Netherlands
}

\section{A R T I C L E I N F O}

\section{Article history:}

Received 18 September 2008

Received in revised form 25 June 2009

Accepted 26 June 2009

Available online $\mathrm{xxxx}$

Communicated by L.A. Hemaspaandra

\section{Keywords:}

Kolmogorov complexity

Compressibility

Time-bounded incompressibility

Barzdins's lemma

Finite strings and infinite sequences

Computational complexity

\begin{abstract}
A B S T R A C T
For every total recursive time bound $t$, a constant fraction of all compressible (low Kolmogorov complexity) strings is $t$-bounded incompressible (high time-bounded Kolmogorov complexity); there are uncountably many infinite sequences of which every initial segment of length $n$ is compressible to $\log n$ yet $t$-bounded incompressible below $\frac{1}{4} n-\log n$; and there is a countably infinite number of recursive infinite sequences of which every initial segment is similarly $t$-bounded incompressible. These results and their proofs are related to, but different from, Barzdins's lemma.
\end{abstract}

(C) 2009 Elsevier B.V. All rights reserved.

\section{Introduction}

Informally, the Kolmogorov complexity of a finite binary string is the length of the shortest string from which the original can be losslessly reconstructed by an effective general-purpose computer such as a particular universal Turing machine $U$. Hence it constitutes a lower bound on how far a lossless compression program can compress. Formally, the conditional Kolmogorov complexity $C(x \mid y)$ is the length of the shortest input $z$ such that the universal Turing machine $U$ on input $z$ with auxiliary information $y$ outputs $x$. The unconditional Kolmogorov complexity $C(x)$ is defined by $C(x \mid \epsilon)$ where $\epsilon$ is the empty string (of length 0 ). Let $t$ be a total recursive function. Then, the

\footnotetext{
* Corresponding author at: Centrum voor Wiskunde en Informatica, Science Park 123, 1098 XG Amsterdam, The Netherlands.

E-mail addresses: egdaylight@yahoo.com (E.G. Daylight), W.M.Koolen-Wijkstra@cwi.nl (W.M. Koolen), Paul.Vitanyi@cwi.nl (P.M.B. Vitányi).

1 a.k.a. Karel van Oudheusden.
}

time-bounded conditional Kolmogorov complexity $C^{t}(x \mid y)$ is the length of the shortest input $z$ such that the universal Turing machine $U$ on input $z$ with auxiliary information $y$ outputs $x$ within $t(n)$ steps where $n$ is the length in bits of $x$. The time-bounded unconditional Kolmogorov complexity $C^{t}(x)$ is defined by $C^{t}(x \mid \epsilon)$. For an introduction to the definitions and notions of Kolmogorov complexity (algorithmic information theory) see [3].

\subsection{Related work}

Already in $1968 \mathrm{~J}$. Barzdins [2] obtained a result known as Barzdins's lemma, probably the first result in resourcebounded Kolmogorov complexity, of which the lemma below quotes the items that are relevant here. Let $\chi$ denote the characteristic sequence of an arbitrary recursively enumerable (r.e.) subset $A$ of the natural numbers. That is, $\chi$ is an infinite sequence $\chi_{1} \chi_{2} \ldots$ where bit $\chi_{i}$ equals 1 if and only if $i \in A$. Let $\chi_{1: n}$ denote the first $n$ bits of $\chi$, and let $C\left(\chi_{1: n} \mid n\right)$ denote the conditional Kolmogorov complexity of $\chi_{1: n}$, given the number $n$. 


\section{Lemma 1.}

(i) For every characteristic sequence $\chi$ of a r.e. set $A$ there exists a constant $c$ such that for all $n$ we have $C\left(\chi_{1: n} \mid n\right) \leqslant$ $\log n+c$.

(ii) There exists a r.e. set $A$ with characteristic sequence $\chi$ such that for every total recursive function $t$ there is a constant $c_{t}$ with $0<c_{t}<1$ such that for all $n$ we have $C^{t}\left(\chi_{1: n} \mid n\right) \geqslant$ $c_{t} n$.

Barzdins actually proved this statement in terms of D.W. Loveland's version of Kolmogorov complexity [4], which is a slightly different setting. He also proved that there is a r.e. set such that its characteristic sequence $\chi=\chi_{1} \chi_{2} \ldots$ satisfies $C\left(\chi_{1: n}\right) \geqslant \log n$ for every $n$. Kummer [5], Theorem 3.1, solving the open problem in Exercise 2.59 of the first edition of [3] proved that there exists a r.e. set such that its characteristic sequence $\zeta=\zeta_{1}, \zeta_{2}, \ldots$ satisfies $C\left(\zeta_{1: n}\right) \geqslant 2 \log n-c$ for some constant $c$ and infinitely many $n$.

The converse of item (i) does not hold. To see this, consider a sequence $\chi=\chi_{1} \chi_{2} \ldots$ and a constant $c^{\prime} \geqslant 2$, such that for every $n$ we have $C\left(\chi_{1: n} \mid n\right) \geqslant n-c^{\prime} \log n$. By item (i), $\chi$ cannot be the characteristic sequence of a r.e. set. Transform $\chi$ into a new sequence $\zeta=\chi_{1} \alpha_{1} \chi_{2} \alpha_{2} \ldots$ with $\alpha_{i}=0^{2^{i}}$, a string of 0 s of length $2^{i}$. While obviously $\zeta$ cannot be the characteristic sequence of a r.e. set, there is a constant $c$ such that for every $n$ we have that $C\left(\zeta_{1: n} \mid n\right) \leqslant \log n+c$.

Item (i) is easy to prove and item (ii) is hard to prove. Putting items (i) and (ii) together, there is a characteristic sequence $\chi$ of a r.e. set $A$ whose initial segments are both logarithmic compressible and timebounded linearly incompressible, for every total recursive time bound. Below, we identify the natural numbers with finite binary strings according to the pairing $(\epsilon, 0)$, $(0,1),(1,2),(00,3),(01,4), \ldots$, where $\epsilon$ again denotes the empty string.

\subsection{Present results}

Theorem 1. Let $k_{0}, k_{1}$ be positive integer constants and $t$ a total recursive function.

(i) A constant fraction of all strings $x$ of length $n$ with $C(x \mid n) \leqslant$ $k_{0} \log n$ satisfies $C^{t}(x \mid n) \geqslant n-k_{1}$ (Lemma 2$)$.

(ii) Let $t(n) \geqslant c n$ for $c>1$ sufficiently large. A constant fraction of all strings $x$ of length $n$ with $C(x \mid n) \leqslant k_{0} \log n$ satisfies $C^{t}(x \mid n) \leqslant k_{0} \log n$ (Lemma 3$)$.

(iii) There exist uncountably many (actually $2^{\aleph_{0}}$ ) infinite binary sequences $\omega$ such that $C\left(\omega_{1: n} \mid n\right) \leqslant \log n$ and $C^{t}\left(\omega_{1: n} \mid n\right) \geqslant \frac{1}{4} n-\log n$ for every $n$; moreover, there exist a countably infinite number of (that is $\aleph_{0}$ ) recursive infinite binary sequences $\omega$ (hence $\left.C\left(\omega_{1: n} \mid n\right)=O(1)\right)$ such that $C^{t}\left(\omega_{1: n} \mid n\right) \geqslant \frac{1}{4} n-\log n$ for every $n$ (Lemma 5$)$.

Note that the order of quantification in Barzdins's lemma is "there exists a r.e. set such that for every total recursive function $t$ there exists a constant $c_{t}$." In contrast, in item (iii) we prove "there is a positive constant such that for every total recursive function $t$ there is a sequence $\omega$." While Barzdins's lemma proves the existence of a single characteristic sequence of a r.e. set that is timelimited linearly incompressible, in item (iii) we prove the existence of uncountably many sequences that are logarithmically compressible over the initial segments, and the existence of a countably infinite number of recursive sequences, such that all those sequences are time-limited linearly incompressible.

We generalize item (i) in Corollaries 1 and 2. Section 2 presents preliminaries. Section 3 gives the results on finite strings. Section 4 gives the results on infinite sequences. Finally, conclusions are presented in Section 5. The proofs for the results are different from Barzdins's proofs.

\section{Preliminaries}

A (binary) program is a concatenation of instructions, and an instruction is merely a string. Hence, we may view a program as a string. A program and a Turing machine (or machine for short) are used synonymously. The length in bits of a string $x$ is denoted by $|x|$. If $m$ is a natural number, then $|m|$ is the length in bits of the $m$ th binary string in length-increasing lexicographic order, starting with the empty string $\epsilon$. We also use the notation $|S|$ to denote the cardinality of a set $S$.

Consider a standard enumeration of all Turing machines $T_{1}, T_{2}, \ldots$. Let $U$ denote a universal Turing machine such that for every $y \in\{0,1\}^{*}$ and $i \geqslant 1$ we have $U(i, y)=$ $T_{i}(y)$. That is, for all finite binary strings $y$ and every machine index $i \geqslant 1$, we have that $U$ 's execution on inputs $i$ and $y$ results in the same output as that obtained by executing $T_{i}$ on input $y$. Let $t$ be a total recursive function. Fix $U$ and define that $C(x \mid y)$ equals $\min _{p}\{|p|: p \in$ $\{0,1\}^{*}$ and $\left.U(p, y)=x\right\}$. For the same fixed $U$, define that $C^{t}(x \mid y)$ equals $\min _{p}\left\{|p|: p \in\{0,1\}^{*}\right.$ and $U(p, y)=x$ in $t(|x|)$ steps $\}$. (By definition the sets over which is minimized are countable and not empty.)

\section{Finite strings}

Lemma 2. Let $k_{0}, k_{1}$ be positive integer constants and $t$ be a total recursive function. There is a positive constant $c_{t}$ such that for sufficiently large $n$ the strings $x$ of length $n$ satisfying $C^{t}(x \mid n) \geqslant n-k_{1}$ form a $c_{t}$-fraction of the strings $y$ of length $n$ satisfying $C(y \mid n) \leqslant k_{0} \log n$.

Proof. The proof is by diagonalization. We use the following algorithm with inputs $t, n, k_{1}$ and a natural number $m$.

\section{Algorithm $\mathcal{A}\left(t, n, k_{1}, m\right)$}

Step 1. Using the universal reference Turing machine $U$, recursively enumerate a finite list of all binary programs $p$ of length $|p|<n-k_{1}$. There are at most $2^{n} / 2^{k_{1}}-1$ such programs. Execute each of these programs on input $n$. Consider the set of all programs that halt within $t(n)$ steps and which output precisely $n$ bits. Call the set of these outputs $B$. Note that $|B| \leqslant 2^{n} / 2^{k_{1}}-1$ and it can be computed in time $O\left(2^{n} t(n) / 2^{k_{1}}\right)$ 
Step 2. Output the $(m+1)$ th string of length $n$, say $x$, in the lexicographic order of all strings in $\{0,1\}^{n} \backslash B$ and halt. If there is no such string then halt with output $\perp$. End of Algorithm

Because of the selection process in Step $1,\left|\{0,1\}^{n} \backslash B\right| \geqslant$ $2^{n}-2^{n} / 2^{k_{1}}+1$ and every $x \in\{0,1\}^{n} \backslash B$ has time-bounded complexity

$C^{t}(x \mid n) \geqslant n-k_{1}$.

For $|m| \leqslant k_{0} \log n-c$, where the constant $c$ is defined below, and provided $\{0,1\}^{n} \backslash B$ is sufficiently large, that is,

$n^{k_{0}} / 2^{c} \leqslant 2^{n}\left(1-\frac{1}{2^{k_{1}}}\right)+1$,

there are at least $n^{k_{0}} / 2^{c}$ strings $x$ of length $n$ that will be output by the algorithm. Call this set $D$. Each string $x \in D$ satisfies

$C\left(x \mid t, n, k_{1}, \mathcal{A}, p\right) \leqslant|m| \leqslant k_{0} \log n-c$.

Since we can describe the fixed $t, k_{0}, k_{1}, \mathcal{A}$, a program $p$ to reconstruct $x$ from these data, and the means to tell them apart, in an additional constant number of bits, say $c$ bits (in this way the quantity $c$ can be deduced from the conditional), it follows that $C(x \mid n) \leqslant k_{0} \log n$. For given $k_{0}, k_{1}$, and $c$, inequality (2) holds for every sufficiently large $n$. For such sufficiently large $n$, the cardinality of the set of strings of length $n$ satisfying both $C(x \mid n) \leqslant k_{0} \log n$ and $C^{t}(x \mid n) \geqslant n-k_{1}$ is at least $|D|=n^{k_{0}} / 2^{c}$. Since the number of strings $x$ of length $n$ satisfying $C(x \mid n) \leqslant k_{0} \log n$ is at most $\sum_{i=0}^{k_{0} \log n} 2^{i}<2 n^{k_{0}}$, the lemma follows with $c_{t}=$ $1 / 2^{c+1}$.

Corollary 1 . Let $k_{0}$ be a positive integer constant and $t$ be a total recursive function. For every sufficiently large natural number $n$, the set of strings $x$ of length $n$ such that $C^{t}(x \mid n) \not k_{0} \log n$ is a positive constant fraction of the strings $y$ of length $n$ satisfying $C(y \mid n) \leqslant k_{0} \log n$.

We can generalize Lemma 2. Let $t$ be a total recursive function, and $f, g$ be total recursive functions such that (4) below is satisfied.

Corollary 2. For every sufficiently large natural number $n$, the set of strings $x$ of length $n$ that satisfy both $C(x \mid n) \leqslant f(n)$ and $C^{t}(x \mid n) \geqslant g(n)$ is a positive constant fraction of the strings $y$ of length $n$ satisfying $C(y \mid n) \leqslant f(n)$.

Proof. Use a similar algorithm $\mathcal{A}(t, n, g, m)$ with $|p|<$ $g(n)$ in Step 1 , and $|m| \leqslant f(n)-c$ in the analysis. Require

$2^{f(n)-c} \leqslant 2^{n}-2^{g(n)}+1$.

Lemma 3. Let $t$ be a total recursive function with $t(n) \geqslant c n$ for some $c>1$ and $k_{0}$ be a positive integer constant. For every sufficiently large natural number $n$, there is a positive constant $c_{t}$ such that the set of strings $x$ of length $n$ satisfying $C^{t}(x \mid n) \leqslant k_{0} \log n$ is a $c_{t}$-fraction of the set of strings $y$ of length $n$ satisfying $C(y \mid n) \leqslant k_{0} \log n$.
Proof. We use the following algorithm that takes positive integers $n, m$ as inputs and computes a string $x$ of length $n$ satisfying $C^{t}(x \mid n) \leqslant k_{0} \log n-c$.

\section{Algorithm $\mathcal{B}(\boldsymbol{n}, \boldsymbol{m})$.}

Output the string $0^{n-|m+1|}(m+1)$ (where $|m+1|$ is the length of the string representation of $m+1)$ and halt. End of Algorithm

Let $k_{0}$ be a positive integer and $c$ a positive integer constant chosen below. Consider strings $x$ that are output by algorithm $\mathcal{B}$ and that satisfy $C^{t}(x \mid n, \mathcal{B}, p) \leqslant|m| \leqslant$ $k_{0} \log n-c$ with $c$ the number of bits to contain descriptions of $\mathcal{B}$ and $k_{0}$, a program $p$ to reconstruct $x$ from these data, and the means to tell the constituent items apart. Hence, $C^{t}(x \mid n) \leqslant k_{0} \log n$. The running time of algorithm $\mathcal{B}$ is $t(n)=O(n)$, since the output strings are length $n$ and to output the $m$ th string with $m \leqslant 2^{k_{0} \log n-c}$ we simply take the binary representation of $m$ and pad it with nonsignificant 0 s to length $n$. Obviously, the strings that satisfy $C^{t}(x \mid n) \leqslant k_{0} \log n$ are a subset of the strings that satisfy $C(x \mid n) \leqslant k_{0} \log n$. There are at least $n^{k_{0}} / 2^{c}$ strings of the first kind while there are at most $2 n^{k_{0}}$ strings of the second kind. Setting $c_{t}=1 / 2^{c+1}$ finishes the proof.

It is well known that if we flip a fair coin $n$ times, that is, given $n$ random bits, then we obtain a string $x$ of length $n$ with Kolmogorov complexity $C(x \mid n) \geqslant n-c$ with probability at least $1-2^{-c}$. Such a string $x$ is algorithmically random. We can also get by with less random bits to obtain resource-bounded algorithmic randomness from compressible strings.

Lemma 4. Let $a, b$ be constants as in the proof below. Given the set of strings $x$ of length $n$ satisfying $C(x \mid n) \leqslant k_{0} \log n$, a total recursive function $t$, the constant $k_{1}$ as before, and $O(a b \log n)$ fair coin flips, we obtain a set of $O(a b)$ strings of length $n$ such that with probability at least $1-1 / 2^{b}$ one string $x$ in this set satisfies $C^{t}(x \mid n) \geqslant n-k_{1}$.

Proof. By Lemma 2, a $c_{t}$ th fraction of the set $A$ of strings $x$ of length $n$ that have $C(x \mid n) \leqslant k_{0} \log n$ also have $C^{t}(x \mid n) \geqslant$ $n-k_{1}$. Therefore, by choosing, uniformly at random, a constant number $a$ of strings from the set $A$ we increase (e.g. by means of a Chernoff bound [3]) the probability that (at least) one of those strings cannot be compressed below $n-k_{1}$ in time $t(n)$ to at least $\frac{1}{2}$. To choose any one string from $A$ requires $O(\log n)$ random bits by dividing $A$ in two equal size parts and repeating this with the chosen half, and so on. The selected $a$ elements take $O(a \log n)$ random bits. Applying the previous step $b$ times, the probability that at least one of the $a b$ chosen strings cannot be compressed below $n-k_{1}$ bits in time $t(n)$ is at least $1-1 / 2^{b}$.

\section{From finite strings to infinite sequences}

We prove a result reminiscent of Barzdins's lemma, Lemma 1. In Barzdins's version, characteristic sequences $\omega$ 
of r.e. sets are considered which by Lemma 1 have complexity $C\left(\omega_{1: n} \mid n\right) \leqslant \log n+c$. Here, we consider a wider class of sequences of which the initial segments are logarithmically compressible (such sequences are not necessarily characteristic sequences of r.e. sets as explained in Section 1.1).

\section{Lemma 5. Let $t$ be a total recursive function.}

(i) There are uncountably many (actually $2^{\aleph_{0}}$ ) sequences $\omega=$ $\omega_{1} \omega_{2} \ldots$ such that both $C\left(\omega_{1: n} \mid n\right) \leqslant \log n$ and $C^{t}\left(\omega_{1: n} \mid n\right)$ $\geqslant \frac{1}{4} n-\log n$ for every $n$.

(ii) The set in item (i) contains a countably infinite number of (that is $\aleph_{0}$ ) recursive sequences $\omega=\omega_{1} \omega_{2} \ldots$ such that $C^{t}\left(\omega_{1: n} \mid n\right) \geqslant \frac{1}{4} n-\log n$ for every $n$.

Proof. (i) Let $g(n)=\frac{1}{2} n-\log n$. Let $c \geqslant 2$ be a constant to be chosen later, $m_{i}=c 2^{i}, B(i), C(i), D(i) \subseteq\{0,1\}^{m_{i}}$ for $i=0,1, \ldots$, and $C(-1)=\{\epsilon\}$. The $C$ sets are constructed so that they contain the target strings in the form of a binary tree, where $C(i)$ contains all target strings of length $m_{i}$. The $B(i)$ sets correspond to forbidden prefixes of length $m_{i}$. The $D(i)$ sets consist of the set of strings of length $m_{i}$ with prefixes in $C(i-1)$ from which the strings in $C(i)$ are selected.

\section{Algorithm $\mathcal{C}(t, g)$.}

for $i:=0,1, \ldots$ do

Step 1. Using the universal reference Turing machine $U$, recursively enumerate the finite list of all binary programs $p$ of length $|p|<g\left(m_{i}\right)$ with $m_{i}=c 2^{i}$ and the constant $c$ defined below. There are at most $2^{g\left(m_{i}\right)}-1$ such programs. Execute each of these programs on all inputs $m_{i}+j$ with $0 \leqslant j<m_{i}$. Consider the set of all programs with input $m_{i}+j$ that halt with output $x=y z$ within $t(|x|)$ time with $|x|=m_{i}+j, y \in$ $C(i-1)$ (then $|y|=m_{i-1}$ for $i>0$ and $|y|=0$ for $i=0$ ), and $z$ is a binary string such that $x$ satisfies $m_{i} \leqslant|x|<m_{i+1}$. There are at most $m_{i}\left(2^{g\left(m_{i}\right)}-1\right)$ such $x$ 's. Let $B(i)$ be the set of the $m_{i}$-length prefixes of these $x^{\prime}$ s. Then, $|B(i)| \leqslant m_{i}\left(2^{g\left(m_{i}\right)}-1\right)$ and it can be computed in time $O\left(m_{i} 2^{g\left(m_{i}\right)} t\left(m_{i+1}\right)\right)$. Note that if $u \in\{0,1\}^{m_{i}} \backslash B(i)$ then $C^{t}(u w|| u w \mid) \geqslant g(|u|)$ for every $w$ such that $|u w|<m_{i+1}$.

Step 2. Let $C(i-1)=\left\{x_{1}, x_{2}, \ldots, x_{h}\right\}$ and $D(i)=$ $\left(C(i-1)\{0,1\}^{*} \cap\{0,1\}^{m_{i}}\right) \backslash B(i)$. for $l:=1, \ldots, h$ do for $k:=0,1$ do put the $k$ th string with initial segment $x_{l}$, in the lexicographic order of $D(i)$, in $C(i)$. If there is no such a string then halt with output $\perp$. od od od End of Algorithm

Clearly, $C(i)\{0,1\}^{*} \subseteq C(i-1)\{0,1\}^{*}$ for every $i=0,1, \ldots$ Therefore, if

$\bigcap_{i=0}^{\infty} C(i)\{0,1\}^{\infty} \neq \emptyset$,

then the elements of this intersection constitute the infinite sequences $\omega$ in the statement of the lemma.
Claim 1. With $g\left(m_{i}\right)=\frac{1}{2} m_{i}-\log m_{i}$, we have $|C(i)|=2^{i+1}$ for $i=0,1, \ldots$.

Proof. The proof is by induction. Recall that $m_{i}=c 2^{i}$ with the constant $c \geqslant 2$.

Base case: $|C(0)|=2$ since $C(-1)=\{\epsilon\}$ and $|D(0)| \geqslant$ $2^{m_{0}}-m_{0}\left(2^{g\left(m_{0}\right)}-1\right) \geqslant 2$.

Induction: Assume that the lemma is true for every $0 \leqslant j<i$. Then, every string in $C(i-1)$ has two extensions in $C(i)$, since for every string in $C(i-1)$ there are $2^{m_{i}-m_{i-1}}$ extensions available of which at most $|B(i)| \leqslant$ $m_{i}\left(2^{g\left(m_{i}\right)}-1\right)$ are forbidden. Namely, $2^{m_{i}-m_{i-1}}-|B(i)| \geqslant$ $2^{m_{i} / 2}-2^{g\left(m_{i}\right)+\log m_{i}}+m_{i} \geqslant 2$. Hence it follows that the binary $k$-choice can always be made in Step 2 of the algorithm for every $l$. Therefore $|C(i)|=2^{i+1}$.

Let a constant $c_{1}$ account for the constant number of bits to specify the functions $t, g$, the algorithm $\mathcal{C}$, and a reconstruction program that executes the following: We can specify every initial $m_{i}$-length segment of a particular $\omega$ in the set on the left-hand side of (5) by running the algorithm $\mathcal{C}$ using the data represented by the $c_{1}$ bits, $m_{i}$, and the indexes $k_{j} \in\{0,1\}$ of the strings in $D(j)$ with initial segment in $C(j-1), 0 \leqslant j \leqslant i$, that form a prefix of $\omega$. Therefore,

$C\left(\omega_{1: m_{i}} \mid m_{i}\right) \leqslant c_{1}+i+1$.

Setting $c=2^{c_{1}+1}$ yields $C\left(\omega_{1: m_{i}} \mid m_{i}\right) \leqslant \log c+i=\log m_{i}$. By the choice of $B(i)$ in the algorithm we know that $C^{t}\left(\omega_{1: m_{i}+j} \mid m_{i}+j\right) \geqslant g\left(m_{i}\right)$ for every $j$ satisfying $0 \leqslant$ $j<m_{i}$. Because $2 m_{i}=m_{i+1}$, for every $n$ satisfying $m_{i} \leqslant$ $n<m_{i+1}, \quad C^{t}\left(\omega_{1: n} \mid n\right) \geqslant \frac{1}{2} m_{i}-\log m_{i} \geqslant \frac{1}{4} n-\log n$. Since this holds for every $i=0,1, \ldots$, item (i) is proven with $C^{t}\left(\omega_{1: n} \mid n\right) \geqslant \frac{1}{4} n-\log n$ for every $n$. The number of $\omega$ 's concerned equals the number of paths in an infinite complete binary tree, that is, $2^{\aleph_{0}}$.

(ii) This is the same as item (i) except that we always take, for example, $k_{i}=0$ (no binary choice) in Step 2 of the algorithm. In fact, we can specify an arbitrary computable $0-1$ valued function to choose the $k_{i}$ 's. There are a countably infinite number of (that is $\aleph_{0}$ ) such functions. The specification of every such function $\phi$ takes $C(\phi)$ bits. Hence we do not have to specify the successive $k_{i}$ bits, and $C\left(\omega_{1: n} \mid n\right)=c_{1}+1+C(\phi)=O(1)$ with $c_{1}$ the constant in the proof of item (i). Trivially, still $C^{t}\left(\omega_{1: m_{i}+j} \mid m_{i}+j\right) \geqslant$ $g\left(m_{i}\right)$ for every $j$ satisfying $0 \leqslant j<m_{i}$. Since this holds for every $i=0,1, \ldots$, item (ii) is proven by item (i).

\section{Conclusions}

We have proved the items promised in the abstract. In Lemma 5 we iterated the proof method of Lemma 2 to prove a result which is reminiscent of Barzdins's Lemma 1, relating compressibility and time-bounded incompressibility of infinite sequences in another manner. Alternatively, we could have studied space-bounded incompressibility. It is easily verified that the results also hold when the time-bound $t$ is replaced by a space bound $s$ and the timebounded Kolmogorov complexity is replaced by spacebounded Kolmogorov complexity. 


\section{Acknowledgement}

We thank the referees for comments, references, pointing out an error in the original proof of Lemma 2 and that the argument used there is both independent and close to that used to prove Theorem 3.2 in [1].

\section{References}

[1] L. Antunes, L. Fortnow, D. van Melkebeek, N.V. Vinodchandran,
Computational depth: Concept and applications, Theoret. Comput. Sci. 354 (3) (2006) 391-404.

[2] Ja.M. Barzdins, Complexity of programs to determine whether natural numbers not greater than $n$ belong to a recursively enumerable set, Soviet Math. Dokl. 9 (1968) 1251-1254.

[3] M. Li, P.M.B. Vitányi, An Introduction to Kolmogorov Complexity and Its Applications, third edition, Springer-Verlag, New York, 2008.

[4] D.W. Loveland, A variant of the Kolmogorov concept of complexity, Inform. and Control 15 (1969) 510-526.

[5] M. Kummer, Kolmogorov complexity and instance complexity of recursively enumerable sets, SIAM J. Comput. 25 (1996) 1123-1143. 